Our data
suggest that
one needs to
be cautious in
targeting the
TGF- $\beta$ pathway
in chronic liver
disease

$\Rightarrow$ LIVER CANCER

\title{
TGF- $\beta$ and cholangiocarcinoma
}

A new study published in

Gastroenterology has further elucidated the function of epithelial transforming growth factor $\beta$ (TGF- $\beta$ ) signalling in the liver. Deletion of TGF- $\beta$ in hepatocytes or cholangiocytes led to increased cholangiocyte proliferation and formation of cholangiocarcinoma. "We tried to understand the role of epithelial TGF- $\beta$ signalling in chronic liver disease-focusing on liver fibrosis, as well as the development of liver cancer because the role of epithelial TGF- $\beta$ signalling was largely unknown," says corresponding author Robert Schwabe. To study this signalling pathway, the authors selectively deleted TGF- $\beta$ receptor 2 (Tgfbr 2 ) in either hepatocytes, cholangiocytes, or both, in mice. Mice were then treated with carbon tetrachloride to induce toxic fibrosis, and had Mdr2 deleted or underwent bile duct ligation for cholestatic liver fibrosis. To induce cancer, Pten was deleted or the mice were treated with diethylnitrosamine. No difference was found in fibrosis, or liver and cholestatic injury between those with no TGF- $\beta$ signalling in hepatocytes and cholangiocytes compared with control mice. Neither was there a difference in the number or size of hepatocellular carcinomas between groups with or without TGF- $\beta$ signalling in the models of liver injury.

All the double-knockout mice (Tgfbr2 and Pten) developed tumours and died at 5-7 months of age, and had severe cholestatic liver disease at the time of death. Based on the expression of biomarkers, the tumours were identified as cholangiocarcinomas. The data suggests that TGF- $\beta$ protects against the development of this type of cancer.
In this model, the authors also observed an increase in the proliferation of cholangiocytes, but not hepatocytes, suggesting that TGF- $\beta$ signalling restricts the proliferation of cholangiocytes. "Our data suggest that one needs to be cautious in targeting the TGF- $\beta$ pathway in chronic liver disease. While this may suppress fibrosis, it may also increase the risk of some forms of liver cancer such as cholangiocarcinoma," remarks Schwabe.

Future studies will aim to better understand the differences in TGF- $\beta$ signalling between cholangiocytes and hepatocytes in liver disease and cancer.

Gillian Patman

ORIGINAL ARTICLE Xueru, M. et al. Epithelial transforming growth factor- $\beta$ signaling doe not contribute to liver fibrosis but protects mice from cholangiocarcinoma. Gastroenterology http://dx.doi.org/101053/j.gastro.2015.11.039 